\title{
ON THE PROPERTIES OF SPECTRAL LAGS AND PEAK-COUNT RATES OF RHESSI GAMMA-RAY BURSTS
}

\author{
J. Řípa ${ }^{1}$, A. Mészáros ${ }^{2}$, P. Veres ${ }^{3}$ and I.H. Park ${ }^{1}$
}

\begin{abstract}
A sample of 427 gamma-ray bursts (GRBs) was observed by the RHESSI satellite in Feb. 2002 - Apr. 2008. We calculated spectral lags and peak-count rates for the first time and constructed a new observational database. This database is statistically studied completing an earlier analysis of durations and hardness ratios. First, we discuss properties of short-, intermediate-, and long-duration GRBs in terms of peak-count rates and spectral lags. Second, we investigate the number of GRB groups using model-based clustering method together with Kolmogorov-Smirnov (K-S) and Anderson-Darling (A-D) tests. The results are: The inter. bursts have properties similar to short bursts. The groups of inter. and long bursts appear to be different. The inter. GRBs in the RHESSI and Swift databases seem to be different phenomena. This work is summary of Rípa et al. (2012).
\end{abstract}

\section{Data sample and methods}

Several clasifications of GRBs have been done, e.g. by Horváth et al. (2006) or Minaev et al. (2010). In our work we fitted two and three lognormal functions to the distribution of durations and hardnesses of RHESSI sample of 427 GRBs and applied maximum likelihood (ML) ratio test. A group of intermediate-duration busts has been found at significance level of 0.3\% (Řípa et al. 2009, 2012).

The spectral lags, i.e. delay between low-energy (25-120 keV) and high-energy (400-1500 keV) counts in GRB light curves, and peak-count rates were calculated. The spectral lags were obtained by fitting the cross-correlation function (in a narrow interval around its peak) of background-subtracted count light curves at these two channels and derived at a time interval covering whole GRB. The properties

\footnotetext{
1 Institute of Basic Science, Sungkyunkwan University, Suwon, South Korea; e-mail: ripa.jakub@gmail.com

2 Astronomical Institute of the Charles University, Prague, Czech Republic

${ }^{3}$ Department of Astronomy and Astrophysics, Penn. State University, State College, USA
} 
Table 1. Left part: results from the A-D tests of spectral lags. The null hypothesis is that two samples are drawn from the same distribution. $P$ is the P-value of the test. Right part: the means, medians and standard deviations $\sigma$ are listed.

\begin{tabular}{cc}
\hline \hline Groups & $\begin{array}{c}\mathrm{A}-\mathrm{D} \mathrm{P} \\
(\%)\end{array}$ \\
\hline Inter.-Short & 16.8 \\
Inter.-Long & 4.2 \\
Short-Long & $<10^{-3}$ \\
\hline
\end{tabular}

\begin{tabular}{cccc}
\hline \hline Group & $\begin{array}{c}\text { Mean } L \\
(\mathrm{~ms})\end{array}$ & $\begin{array}{c}\text { Median } L \\
(\mathrm{~ms})\end{array}$ & $\begin{array}{c}\sigma \\
(\mathrm{ms})\end{array}$ \\
\hline Short & 4.9 & 1.9 & 16.7 \\
Inter. & 28.7 & 5.9 & 78.4 \\
Long & 178.0 & 50.8 & 874.9 \\
\hline
\end{tabular}

of the three identified clusters of GRBs were studied by K-S and A-D tests (see Tables 1 and 2). Next a model-based clustering technique was applied on three variables: $T_{90}$ durations, hardnesses $H$, and peak-count rates $F$. The Bayesian Information Criterion (BIC) was used (Table 3). The function which is maximized is: $\mathrm{BIC}=2 \ln l_{\max }-m \ln N$, where $l_{\max }$ is the ML of the model, $m$ is the number of free parameters, and $N$ is the size of the sample. Surprisingly the group of long bursts is now separated into high- and low-peak flux clusters (see Fig. 1).

Table 2. Left part: results of the K-S test applied on the peak-count rates $F$. The K-S distance $D$ and the K-S significance $P$ are mentioned. Right part: the means, medians, and standard deviations of the peak-count rates are listed.

\begin{tabular}{ccc}
\hline \hline Groups & $D$ & $\begin{array}{c}\text { K-S P } \\
(\%)\end{array}$ \\
\hline Inter.-Short & 0.44 & 0.9 \\
Inter.-Long & 0.55 & $3 \times 10^{-5}$ \\
Short-Long & 0.69 & $<10^{-6}$ \\
\hline
\end{tabular}

\begin{tabular}{cccc}
\hline \hline Group & $\begin{array}{c}\text { Mean } \\
F(\mathrm{cnt} / \mathrm{s})\end{array}$ & $\begin{array}{c}\text { Median } \\
F(\mathrm{cnt} / \mathrm{s})\end{array}$ & $\begin{array}{c}\sigma \\
(\mathrm{cnt} / \mathrm{s})\end{array}$ \\
\hline Short & 9490 & 5160 & 20420 \\
Inter. & 4410 & 2550 & 5590 \\
Long & 2590 & 1040 & 7670 \\
\hline
\end{tabular}

\section{Conclusions}

ML test in the duration-hardness plane of 427 RHESSI GRBs exhibits a third in duration intermediate group at significance level of $0.3 \%$. The spectral lags and peak-count rates were calculated for GRBs observed by the RHESSI satellite for the first time and thus we constructed a new observational database. Then the three GRB clusters were analyzed with respect to the spectral lags and peak-count rates. The difference between short and long groups were confirmed. K-S and A-D tests applied on spectral lags and peak-count rates indicate that the inter. group in the RHESSI database might be a longer tail of the short group or at least has some common properties with this short group. Contrary to this, the inter. and the long groups seem to have different properties. We compared properties of RHESSI inter.-duration busts with those found by Swift (Veres et al. 2010; de Ugarte Postigo et al. 2011). The inter.-duration bursts found in these two databases seem to be represented by different phenomena. 
Table 3. A summary of the results from the model-based clustering.

\begin{tabular}{ccccccccc}
\hline \hline & Model & $k$ & BIC & $\Delta$ BIC & $\Delta$ BIC & $\Delta$ BIC & $\Delta$ BIC & Evidence \\
& & & & $k=1$ & $k=2$ & $k=3$ & $k=4$ & \\
\hline Two param. & EVI & 2 & -681.5 & $>10$ & $\times$ & $>10$ & & Very strong \\
\hline Three param. & EEE & 3 & -1156.6 & & $>10$ & $\times$ & $>10$ & Very strong \\
\hline Four param. & VVV & 2 & -1768.4 & $>10$ & $\times$ & $>10$ & & Very strong \\
\hline
\end{tabular}

Note. - The results for model-based clustering applied on two, three, and four parameters is presented. The values of BIC for the best fitted models with $k$ components are listed, as well as the differences to the models with other number of components. $\Delta \mathrm{BIC}>10$ strongly supports the model with higher BIC. The volumes and shapes of all clusters may be equivalent (E) or may vary $(\mathrm{V})$ and axes of all clusters may be restricted to parallel orientations with the coordinate axes (I).

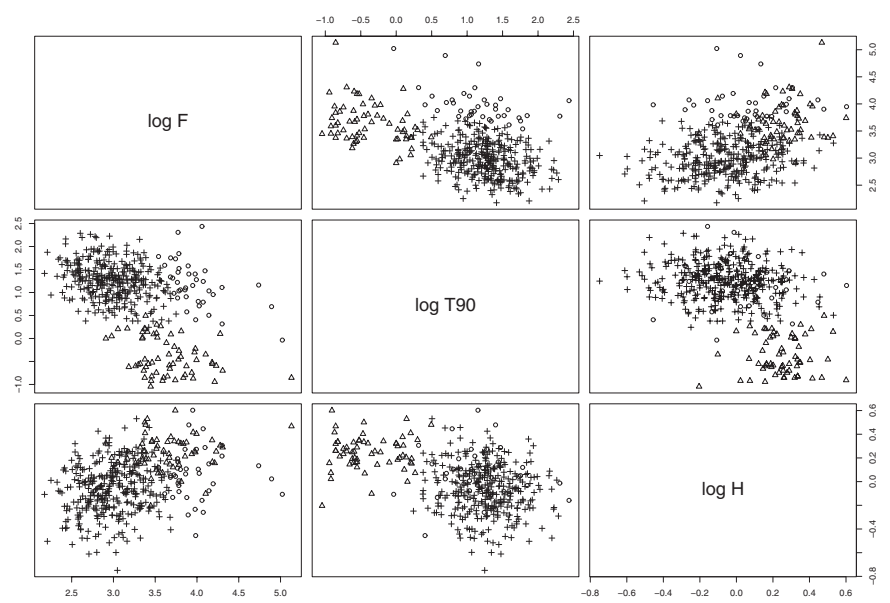

Fig. 1. GRBs with measured $T_{90}, H$, and $F$ assigned to 3 groups by the best fit model (EEE). Triangles, circles, and crosses denote short-duration, long-duration high- and long-duration low-peak count rate GRBs, respectively.

This study was supported by the OTKA grant K77795, by the Grant Agency of the Czech Republic grant No. P209/10/0734, by the Research Program MSM00216-20860 of the Ministry of Education of the Czech Republic, and by Creative Research Initiatives (RCMST) of MEST/NRF.

\section{References}

Horváth, I., Balázs, L.G., Bagoly, Z., et al., 2006, A\&A, 447, 23

Minaev, P.Yu., Pozanenko, A.S., Loznikov, V.M., et al., 2010, Astroph. Bull., 65, 326

Ř́pa, J., Mészáros, A., Wigger, C., et al., 2009, A\&A, 498, 399

Ř́pa, J., Mészáros, A., Veres, P., et al., 2012, ApJ, 756, 44

de Ugarte Postigo, A., Horváth, I., Veres, P., et al., 2011, A\&A, 525, A109

Veres, P., Bagoly, Z., Horváth, I., et al., 2010, ApJ, 725, 1955 
